Vietnam Journal of Mechanics, VAST, Vol.30, No.1 (2008), pp. 11-19

\title{
A UNIFIED KRYLOV-BOGOLIUBOV- MITROPOLSKII METHOD FOR SOLVING HYPERBOLIC-TYPE NONLINEAR PARTIAL DIFFERENTIAL SYSTEMS
}

\author{
M. Zahurul Islam \\ Department of Applied Mathematics, Rajshahi University, Rajshahi-6205, Bangladesh \\ M. Shamsul Alam and M. Bellal Hossain \\ Department of Mathematics, Rajshahi University of Engineering and Technology, \\ Rajshahi-6204,Bangladesh,Email address: msalam1964@yahoo.com
}

\begin{abstract}
A general asymptotic solution is presented for investigating the transient response of non-linear systems modeled by hyperbolic-type partial differential equations with small nonlinearities. The method covers all the cases when eigen-values of the corresponding unperturbed systems are real, complex conjugate, or purely imaginary. It is shown that by suitable substitution for the eigen-values in the general result that the solution corresponding to each of the three cases can be obtained. The method is an extension of the unified Krylov-Bogoliubov-Mitropolskii method, which was initially developed for un-damped, under-damped and over-damped cases of the second order ordinary differential equation. The methods also cover a special condition of the over-damped case in which the general solution is useless.

Keywords: Unified KBM method, Oscillation, Non-oscillation
\end{abstract}

\section{INTRODUCTION}

Krylov-Bogoliubov-Mitropolskii (KBM) [1, 2] method is one of the widely used techniques to obtain analytical solutions of weakly nonlinear ordinary differential equations. The method was originally developed to find periodic solutions of second-order nonlinear ordinary differential equations. Popov [3] extended the method to damped nonlinear systems. Murty, Deekshatulu and Krisna [4] investigated nonlinear over-damped systems by this method. Murty [5] used their earlier solution [4] as a general solution for un-damped, damped and over-damped cases, which is the basis of the unified theory. Since Murty's technique is a generalization of the KBM method, many authors extended this technique in various oscillatory and non-oscillatory systems. Bojadziev and Edwards [6] investigated nonlinear damped oscillatory and non-oscillatory systems with varying coefficients following Murty's [5] unified method. Recently Shamsul [7, 8] has presented a unified formula to obtain a general solution of an $n$-th order ordinary differential equation with constant and slowly varying coefficients. The KBM method was extended to partial differential equation with small nonlinearities by Mitropolskii and Mosenkov [9]. Bojadziev and Lardner [10] extended the KBM method to hyperbolic-type partial differential equation

$$
\rho(x) u_{t t}=\frac{\partial}{\partial x}\left(\chi(x) u_{x}\right)+\varepsilon f\left(x, u, u_{x}, u_{t}\right),
$$

where the subscript denotes differentiations, $\varepsilon$ is a small parameter and $f$ is a given nonlinear function. 
Bojadziev and Lardner [10] mainly investigated the mono-frequent solution of (1). Bojadziev and Lardner [11] also found mono-frequent type solutions of the partial differential equation with a linear damping effect, $-2 \rho(x) k u_{t}$, of the form

$$
\rho(x)\left(u_{t t}+2 k u_{t}\right)=\frac{\partial}{\partial x}\left(\chi(x) u_{x}\right)+\varepsilon f\left(x, u, u_{x}, u_{t}\right) .
$$

In another recent paper, Shamsul, Akber and Zahurul [12] present a general formula to investigate a class of nonlinear partial differential equations. In this paper a general asymptotic solution of (2) is obtained which covers the over-damped, damped and un-damped cases. Thus, the unified KBM method [5] is independent of whether the unperturbed system has real, complex conjugate, or purely imaginary eigen-values whether described by an ordinary or partial differential equation. Moreover a special over-damped solution is obtain which is essential when the general solution fails to give desired results (See [13] for details).

\section{THE METHOD}

Let us consider that $u(x, t, \varepsilon)$ satisfies a pair of homogeneous boundary conditions involving $u$ and its derivatives at $x=0$ and $x=l$ :

$$
B_{j}(u) \equiv \beta_{j 1} u(0, t)+\beta_{j 2} u_{x}(0, t)+\beta_{j 3} u(l, t)+\beta_{j 4} u_{x}(l, t), \quad j=1,2,
$$

where $\beta_{j r}, j=1,2$ and $r=1,2,3,4$ are eight constants.

The investigation of mono-frequent damped oscillations of equation (2) is of interest in certain problems occurring in mechanics. For instance, such an equation describes the vibration of certain nonlinear elastic system in the presence of strong viscous damping. We shall examine in detail the longitudinal vibrations of a rod. The material of rod is taken to be predominately Hooken but with, in addition, small. nonlinear elastic characteristic.

First of all, we consider the unperturbed system (2)

$$
\rho(x)\left(u_{t t}^{(0)}+2 k u_{t}^{(0)}\right)=\frac{\partial}{\partial x}\left(\chi(x) u_{x}^{(0)}\right),
$$

with boundary conditions (3).

It is well known that with prescribed boundary conditions (3) satisfying certain selfadjoin ness, (4) has a complete set of separable solutions which can be written in the form

$$
\phi_{n}(x) e^{-k t} a_{n, 0} \sinh \left(\omega_{n} t+\dot{\psi}_{n, 0}\right), \quad n=1,2, \cdots
$$

where $a_{n, 0}$ and $\psi_{n, 0}$ are arbitrary constants. The set of functions $\left\{\phi_{n}(x)\right\}, n=1,2, \cdots$ satisfy the ordinary differential equation

$$
\frac{d}{d x}\left(\chi(x) \frac{d \phi_{n}(x)}{d x}\right)+\left(k^{2}-\omega_{n}^{2}\right) \rho(x) \phi_{n}(x)=0, \quad B_{j}\left(\phi_{n}(x)\right)=0, \quad j=1,2 .
$$

In order to solve oscillating processes, Bojadziev and Lardner [11] assumed damping is less than critical, i.e., $\omega_{n}^{2}>0$. Here we remove this restriction and consider more general $\omega_{s}$ such that $\omega_{s}^{2}>0$ or/and $\omega_{s}^{2}<0$. It is to be noted that eigen-values are determined from boundary conditions (3). Let us consider $\left\{\phi_{n}(x)\right\}$ are normalized, so that

$$
\int_{0}^{l} \rho(x) \phi_{m}(\mathrm{x}) \phi_{n}(\mathrm{x}) \mathrm{dx}=\delta_{m, n}
$$


Now we shall find a mono-frequent solution of (2) for which $\varepsilon=0$ corresponds to frequency $\omega_{1}$. Following the KBM method, we look for a solution of the form

$$
u(x, t, \varepsilon)=\phi_{1}(x) a(t) \sinh \psi(t)+\varepsilon u_{1}(x, a, \psi)+\mathrm{O}\left(\varepsilon^{2}\right) \cdots,
$$

where $a$ and $\psi$ satisfy the equations

$$
\dot{a}=-k a+\varepsilon A_{1}(a)+\mathrm{O}\left(\varepsilon^{2}\right) \cdots, \quad \dot{\psi}=\omega_{1}+\varepsilon B_{1}(a)+\mathrm{O}\left(\varepsilon^{2}\right) \cdots .
$$

Substituting (8) into (2), making use of (9) and comparing the coefficients of $\varepsilon$, we obtain

$$
\begin{aligned}
& \rho(x) \phi_{1}(x)\left[\left(2 \omega_{1} A_{1}-k a^{2} \frac{d B_{1}}{d a}\right) \cosh \psi+\left(-k a \frac{d A_{1}}{d a}+k A_{1}+2 \omega_{1} a B_{1}\right) \sinh \psi\right] \\
& +\rho(x)\left[\left(-k a \frac{\partial}{\partial a}+\omega_{1} \frac{\partial}{\partial \psi}\right)^{2}+2 k\left(-k a \frac{\partial}{\partial a}+\omega_{1} \frac{\partial}{\partial \psi}\right)\right) u_{1}=\frac{\partial}{\partial x}\left(\chi(x) \frac{\partial u_{1}}{\partial x}\right]+f^{(0)},
\end{aligned}
$$

where $f^{(0)}=f\left(x, u_{0}, u_{0, x}, u_{0, t}\right)$ and $u_{0}=\phi_{1}(x) a(t) \sinh \psi(t)$.

Let us expand $u_{1}$ as a Fourier series in $x$ using the basis $\left\{\phi_{n}(x)\right\}$

$$
u_{1}(x, a, \psi)=\sum_{j=1}^{\infty} v_{j}(a, \psi) \phi_{j}(x) .
$$

Substituting (11) into (10), multiplying both sides by $\phi_{s}(x)$ and integrating with respect to $x$ within limits from 0 to $l$, and making use of $(6)$ and (7) gives

$$
\begin{aligned}
& \left.\left[\left(2 \omega_{1} A_{1}-k a^{2} \frac{d B_{1}}{d a}\right) \cosh \psi+\left(-k a \frac{d A_{1}}{d a}+k A_{1}+2 \omega_{1} a B_{1}\right) \sinh \psi\right)\right] \delta_{1, s} \\
& +\left[\left(-k a \frac{\partial}{\partial a}+\omega_{1} \frac{\partial}{\partial \psi}+k\right)^{2}-\omega_{s}^{2}\right] v_{s}=F_{s}(a, \psi),
\end{aligned}
$$

where

$$
F_{s}(a, \psi)=\int_{0}^{l} f^{(0)}(x, a, \psi) \ddot{\phi}_{s}(x) d x .
$$

It is customary to solve (12) for unknown functions $A_{1}, B_{1}$ and $v_{s}, s=1,2, \cdots$ under the assumption that $v_{1}$ does not contain fundamental terms involving $\sinh \psi$ and $\cosh \psi$ (see $[4,5]$ for details). It is assumed that $F_{s}$ is expanded as a series of hyperbolic functions $F_{s}=F_{s, 0}(a)+F_{s, 1}(a) \cosh \psi+F_{s, 2}(a) \cosh 2 \psi \cdots+G_{s, 1}(a) \sinh \psi+G_{s, 2}(a) \sinh 2 \psi+\cdots$

It is noted that series (14) becomes a Fourier series when the motion is un-damped or under-damped, i.e., $\omega_{1}$ as well as $\psi, G_{s, 1}, G_{s, 2}$ are purely imaginary.

Substituting the values of $F_{s}$ from (14) into (12) and assuming that $v_{1}$ excludes terms involving $\sinh \psi$ and $\cosh \psi$, we obtain 0

$$
\begin{gathered}
2 \omega_{1} A_{1}-k a^{2} \frac{d B_{1}}{d a}=F_{1,1} \\
-k a \frac{d A_{1}}{d a}+k A_{1}+2 \omega_{1} B_{1}=G_{1,1} \\
{\left[\left(-k a \frac{\partial}{\partial a}+\omega_{1} \frac{\partial}{\partial \psi}+k\right)^{2}-\omega_{1}^{2}\right] v_{1}=F_{1,0}+F_{1,2} \cosh 2 \psi+G_{1,2} \sinh 2 \psi \cdots}
\end{gathered}
$$


and

$$
\left[\left(-k a \frac{\partial}{\partial a}+\omega_{1} \frac{\partial}{\partial \psi}+k\right)^{2}-\omega_{s}^{2}\right] v_{s}=\left(F_{s, 0}+F_{s, 1} \cosh \psi+G_{s, 1} \sinh \psi, s \geq 2 .\right.
$$

The particular solutions of (15)-(18) give unknown functions $A_{1}, B_{1}$ and $v_{s}, s=$ $1,2, \cdots$, which complete the determination of the first order solution of (2). The method can be extended to higher order approximations in a similar way.

\section{EXAMPLE}

As an example of the above procedure we may consider the longitudinal vibration of a nonlinear elastic rod described by equation

$$
\rho u_{t t}+\kappa u_{t}=\sigma_{x},
$$

where $u$ is longitudinal displacement, $\sigma$ axial tension, $\rho$ mass per unit length. The term $2 \kappa u_{t}$ represents viscous damping. The stress-strain relation is assumed as

$$
\sigma=K e+\frac{1}{3} \varepsilon E e^{3}
$$

where $K$ is Young's modulus, $e=u_{x}$ axial strain and the second term containing $E$ represents nonlinear elastic behavior. Eliminating $\sigma$ from (19) and (20) and substituting $\kappa=2 \rho k$, we obtain a partial differential equation in the form

$$
\rho\left(u_{t t}+2 k u_{t}\right)=K u_{x x}+\varepsilon E u_{x}^{2} u_{x x} .
$$

Let us consider the boundary conditions

$$
u(0, t)=0, h u_{x}(l, t)+u(l, t)=0 .
$$

Applying boundary conditions (22), we obtain the eigen-functions and eigen-values of the unperturbed 21) as:

$$
\phi_{n}(x)=c_{n} \sin p_{n} x, \quad \lambda_{n}^{2}=\frac{K p_{n}^{2}}{\rho}, \quad n=1,2, \cdots
$$

where $\left\{p_{n}\right\}$ are the eigen-values of equation

$$
\tan p l=-h p
$$

and constants $\left\{c_{n}\right\}$ satisfy

$$
\frac{\rho c_{n}^{2}}{2}\left(l+\frac{h}{1+h^{2} p_{n}^{2}}\right)=1, \quad n=1,2, \cdots .
$$

In (21), the nonlinear function is $f=E u_{x}^{2} u_{x x}$. Therefore, we have

$$
F_{s}=E_{s} \sinh ^{3} \psi=\frac{1}{4} E_{s} a^{3}(\sinh 3 \psi-3 \sinh \psi)
$$

where $E_{s}=E \int_{0}^{l}\left(\frac{d \phi_{1}}{d x}\right)^{2} \frac{d^{2} \phi_{1}}{d x^{2}} \phi_{s} d x$. Therefore, only non-zero coefficients of $F_{s, n}$ and $G_{s, n}, n=$ $1,2, \cdots$, are $G_{s, 1}=-\frac{3}{4} E_{s} a^{3}$ and $G_{s, 3}=\frac{1}{4} E_{s} a^{3}$. Substituting the values of $G_{s, 1}$ and $G_{s, 3}$ into (15)-(18) and solving them, we obtain

$$
A_{1}=\frac{3 E_{1} k a^{3}}{8\left(k^{2}-\omega_{1}^{2}\right)}, \quad B_{1}=\frac{3 E_{1} \omega_{1} a^{2}}{8\left(k^{2}-\omega_{1}^{2}\right)},
$$




$$
v_{1}=\frac{E_{1} a^{3}\left(3 k \omega_{1} \cosh 3 \psi+\left(k^{2}+2 \omega_{1}^{2}\right) \sinh 3 \psi\right)}{16\left(k^{2}-\omega_{1}^{2}\right)\left(k^{2}-4 \omega_{1}^{2}\right)}, \quad k \neq 2 \omega_{1}
$$

and

$$
\begin{gathered}
v_{s}=\frac{-E_{s} a^{3}\left(4 k \omega \cosh \psi+\left(4 k^{2}+\omega_{s}^{2}-\omega_{1}^{2}\right) \sinh \psi\right)}{4\left(4 k^{2}-\left(\omega_{s}-\omega_{1}\right)^{2}\right)\left(4 k^{2}-\left(\omega_{s}+\omega_{1}\right)^{2}\right)} \\
+\frac{3 E_{s} a^{3}\left(12 k \omega_{1} \cosh 3 \psi+\left(4 k^{2}+\omega_{s}^{2}-9 \omega_{1}^{2}\right) \sinh 3 \psi\right)}{4\left(4 k^{2}-\left(\omega_{s}-3 \omega_{1}\right)^{2}\right)\left(4 k^{2}-\left(\omega_{s}+3 \omega_{1}\right)^{2}\right)}, \quad s \geq 2 .
\end{gathered}
$$

Substituting the values of $A_{1}$ and $B_{1}$ from (27) into (9), we integrate them with respect to $t$, and obtain

$$
a=\frac{a_{0} e^{-k t}}{\sqrt{1-\frac{3 \varepsilon E_{1} a_{0}^{2}\left(1-e^{-2 k t}\right)}{8\left(k^{2}-\omega_{1}^{2}\right)}}}, \quad \psi=\psi_{0}+\omega_{1} t-\frac{\omega_{1}}{2 k} \ln \left(1-\frac{3 \varepsilon E_{1} a_{0}^{2}\left(1-e^{-2 k t)}\right.}{8\left(k^{2}-\omega_{1}^{2}\right)}\right),
$$

Thus the first order solution of (19) is

$$
u(x, t, \varepsilon)=\phi_{1}(x) a \sinh \psi+\varepsilon \sum_{s=1}^{\infty} \phi_{s}(x) v_{s}(a, \psi),
$$

where $a, \psi, v_{1}$ and $v_{s}, s \geq 2$ are given respectively by (30), (28) and (29). In the case of an under-damped system, all $\omega_{s}$ are replaced by $i \omega_{s}, a$ by $-i a, \psi$ by $i \psi, \cosh i \psi$ by $\cos \psi$ and $\sinh i \psi$ by $i \sin \psi$. These yield

$$
u(x, t, \varepsilon)=\phi_{1}(x) a \sin \psi+\varepsilon \sum_{s=1}^{\infty} \phi_{s}(x) v_{s}(a, \psi),
$$

where $a, \psi, v_{1}$ and $v_{s}, s \geq 2$ are given by

$$
\begin{aligned}
& a=a_{0} e^{-k t}\left(\sqrt{1+\frac{3 \varepsilon E_{1} a_{0}^{2}\left(1-e^{-2 k t}\right)}{8\left(k^{2}+\omega_{1}^{2}\right)}}\right)^{-1}, \\
& \psi=\psi_{0}+\omega_{1} t-\frac{\omega_{1}}{2 k} \ln \left(1+\frac{3 \varepsilon E_{1} a_{0}^{2}\left(1-e^{-2 k t)}\right.}{8\left(k^{2}+\omega_{1}^{2}\right)}\right), \\
& v_{1}=-\frac{E_{1} a^{3}\left(3 k \omega_{1} \cos 3 \psi+\left(k^{2}-2 \omega_{1}^{2}\right) \sin 3 \psi\right)}{16\left(k^{2}+\omega_{1}^{2}\right)\left(k^{2}+4 \omega_{1}^{2}\right)}
\end{aligned}
$$

and

$$
\begin{gathered}
v_{s}=\frac{3 E_{s} a^{3}\left(4 k \omega \cos \psi+\left(4 k^{2}+\omega_{s}^{2}-\omega_{1}^{2}\right) \sin \psi\right)}{4\left(4 k^{2}+\left(\omega_{s}-\omega_{1}\right)^{2}\right)\left(4 k^{2}+\left(\omega_{s}+\omega_{1}\right)^{2}\right)} \\
-\frac{E_{s} a^{3}\left(12 k \omega_{1} \cos 3 \psi+\left(4 k^{2}+\omega_{s}^{2}-9 \omega_{1}^{2}\right) \sin 3 \psi\right)}{4\left(4 k^{2}+\left(\omega_{s}-3 \omega_{1}\right)^{2}\right)\left(4 k^{2}+\left(\omega_{s}+3 \omega_{1}\right)^{2}\right)}, \quad s \geq 2 .
\end{gathered}
$$

It is obvious that when $k>0$, the solution is similar to Bojadziev and Lardner [11], and identical to that obtained in [9] when $k=0$. 


\section{A SPECIAL DAMPING CONDITION}

Clearly $v_{1}$ (See (28)) is not defined for $k=2 \omega_{1}$. This situation occurs when the difference of $3 \lambda_{1}$ and $\lambda_{2}$ or $3 \lambda_{2}$ and $\lambda_{1}$ are significant (where $\lambda_{1}$ and $\lambda_{2}$ are the eigen-values of the corresponding unperturbed system (19)). In this case $v_{1}$ must contain a secular type term $t e^{-t}$ (See [13] for details). In this situation we seek a solution of the form [13],

$$
u(x, t, \varepsilon)=\phi_{1}(x)\left(a e^{-\lambda t}+b e^{-\mu t}\right)+\varepsilon u_{1}(x, a, b, t)+\mathrm{O}\left(\varepsilon^{2}\right),
$$

where $a$ and $b$ satisfy the equations

$$
\dot{a}=\varepsilon A_{1}(a, b, t)+\mathrm{O}\left(\varepsilon^{2}\right), \quad \dot{b}=\varepsilon B_{1}(a, b, t)+\mathrm{O}\left(\varepsilon^{2}\right) .
$$

Substituting (36) into (2), utilizing (37) and comparing the coefficients of $\varepsilon$, we obtain

$$
\begin{aligned}
& \left.\rho(x) \phi(x)\left[\left(\frac{\partial A_{1}}{\partial t}-\lambda A_{1}+\mu A_{1}\right) e^{-\lambda t}+\left(\frac{\partial B_{1}}{\partial t}-\lambda B_{1}+\mu B_{1}\right) e^{-\mu t}\right)\right] \\
& +\rho(x)\left(\frac{\partial^{2}}{\partial t^{2}}+2 k \frac{\partial}{\partial t}\right) u_{1}=\frac{\partial}{\partial x}\left(\chi(x) \frac{\partial u_{1}}{\partial x}\right)+f^{0},
\end{aligned}
$$

where $f^{0}=f\left(x, u_{0}, u_{0, x}, u_{0, t}\right)$ and $u_{0}=\phi_{1}(x)\left(a e^{-\lambda t}+b e^{-\mu t}\right)$.

Let us expand $u_{1}$ as a Fourier series in $\mathrm{x}$ using the basis $\left\{\phi_{n}(x)\right\}$ as

$$
u_{1}(x, a, b, t)=\sum_{j=1}^{\alpha} v_{j}(a, b, t) \phi_{j}(x) .
$$

Substituting (39) into (38), multiplying both sides by $\phi_{s}(x)$ and integrating with respect to $x$ within limits 0 to $l$, and making use of (6) and (7) gives

$$
\begin{aligned}
& {\left[\left(\frac{\partial A_{1}}{\partial t}-\lambda A_{1}+\mu A_{1}\right) e^{-\lambda t}+\left(\frac{\partial B_{1}}{\partial t}-\lambda B_{1}+\mu B_{1}\right) e^{-\mu t}\right] \delta_{1, s}} \\
& +\left(\frac{\partial}{\partial t}+\lambda\right)\left(\frac{\partial}{\partial t}+\mu\right) v_{s}=F_{s}(a, b, t),
\end{aligned}
$$

where

$$
F_{s}(a, b, t)=\int_{0}^{l} f^{0}\left(x, u_{0}, u_{0, x}, u_{0, t}\right) \phi_{s}(x) d x .
$$

In general, $F_{s}(a, b, t)$ can be expanded as a Taylor's series

$$
F_{s}(a, b, t)=\sum_{j, r=0} F_{j, r}(a, b) e^{-(j \lambda+r \mu) t} .
$$

In order to determine over-damped solutions of (1), we assume that $u_{1}$ does not contain terms with $e^{-(j \lambda+r \mu) t}$, where $j \lambda+r \mu<k(j+r)$, so that the coefficient of the expansion of $u_{1}$ does not become large, and $u_{1}$ does not contain the secular type term $t e^{-(j \lambda+r \mu) t}$.

The function $F_{s}(a, b, t)$ becomes

$$
F_{s}(a, b, t)=E_{s}\left(a^{3} e^{-3 \lambda t}+3 a^{2} b e^{-(2 \lambda+j) t}+3 a b^{2} e^{-(\lambda+2 \mu) t}+b^{3} e^{-3 \mu t}\right) .
$$


Substituting the values of $F_{s}(a, b, t)$ from (43) into (40) we obtain the following equations

$$
\begin{aligned}
& \left(\frac{\partial A_{1}}{\partial t}-\lambda A_{1}+\mu A_{1}\right)=E_{1} b^{3} e^{-(3 \mu-\lambda) t}, \\
& \left(\frac{\partial B_{1}}{\partial t}-\lambda B_{1}+\mu B_{1}\right)=E_{1} a b^{2} e^{-(\mu+\lambda) t}
\end{aligned}
$$

and

$$
\left(\frac{\partial}{\partial t}+\lambda\right)\left(\frac{\partial}{\partial t}+\mu\right) v_{s}=E_{1}\left(a^{3} e^{-3 \lambda t}+3 a b^{2} e^{-(2 \lambda+\mu) t} .\right.
$$

Solving (44)-(46), we obtain

$$
\begin{aligned}
& A_{1}= \begin{cases}\frac{-E_{1} b^{3} e^{-(-\lambda+3 \mu) t}}{2 \mu(-\lambda+3 \mu)}, & \lambda \neq 3 \mu, \\
\frac{-E_{1} b^{3}}{2 \mu}, & \lambda=3 \mu,\end{cases} \\
& B_{1}=\frac{-E_{1} a b^{2} e^{-(\lambda+\mu) t}}{2 \mu},
\end{aligned}
$$

and

$$
v_{1}=E_{1}\left(\frac{a^{3} e^{-3 \lambda t}}{2 \lambda(3 \lambda-\mu)}+\frac{3 a b^{2} e^{-(2 \lambda+\mu) t}}{(\lambda+\mu)(2 \lambda)}\right) .
$$

Substituting the values of $A_{1}$ and $B_{1}$ from (48) and (49) into (37), and integrating them with respect to $t$, we obtain

$$
\begin{gathered}
a=a_{0}-\frac{E_{1} \varepsilon b_{0}^{3}}{2 \mu(-\lambda+3 \mu)}\left(1-e^{-(-\lambda+3 \mu) t}\right), \quad \lambda \neq 3 \mu, \\
=a_{0}-\frac{E_{1} \varepsilon b_{0}^{3}}{2 \mu} t, \quad \lambda=3 \mu, \\
b=b_{0}-\frac{E_{1} \varepsilon a_{0} b_{0}^{2}}{2 \mu(\lambda+\mu)}\left(1-e^{-(\lambda+\mu) t}\right) .
\end{gathered}
$$

Thus the first order special over-damped solution of (19) is

$$
u(x, t, \varepsilon)=\phi_{1}(x)\left(a e^{-\lambda t}+b e^{-\mu t}+\varepsilon v_{1}\right),
$$

where $a, b$ and $v_{1}$ are given by (50), (51) and (49) respectively.

\section{RESULTS AND DISCUSSION}

A unified solution is found for a nonlinear partial differential equation based on the works of Murty et. al. $[4,5]$. In order to test the accuracy of this unified solution, we compare the solution to the numerical solution (consider to be exact). With regard to such a comparison concerning the presented unified method of this paper, we refer to. a recent work by Shamsul [7] and as well as a previous work by Murty, Deekshatulu and Krisna [4]. Moreover, we compare the perturbation solution to the unperturbed solution to denote the response of the nonlinear term.

The solution (31) is well established and useful as an over-damped solution of (19). We are interested in comparing it with numerical solution (generated by finite difference 
method). Let us consider an over-damped case of (19), in which $\rho=1, k=1.25, l=2$, $K=1$. The solutions to (24) are $1.144465,2.543493,4.048082 \cdots$, eigen-values are $-1.25 \pm$ $0.502693,-1.25 \pm 2.215143 i,-1.25 \pm 3.850256 i \cdots$, and one set of eigen-value is real and $\omega_{1}=0.502693$. For $\varepsilon=0.2$ and initial values $\left[u(x, 0)=0.90667 \sin (1.144465 x), u_{t}(x, 0)=\right.$ 0 ], $u(x, t, \varepsilon)$ has been evaluated and the corresponding numerical solution of (19) has been computed. The results for $x=2$ (i.e. for the lower end of the rod) and $x=1$ (i.e. for the middle point of the rod) are presented respectively in Fig. 1(a) and Fig. 1(b). From the figures it is clear that solution Eq. (31) compares well the numerical solution.

The solution (32) is also well established and useful as an un-damped and under damped solution of (19). Let us consider the un-damped case of (19), in which, $\rho=1$, $k=0.0, l=2, K=1$. The solution of $(24)$ are $1.144465,2.543493,4.048082 \cdots$ or eigen values are $1.144465,2.543493,4.048082 \cdots$ For $\varepsilon=0.2$ and for initial values $[u(x, 0)=$ $\left.0.90667 \sin (1.144465 x), u_{t}(x, 0)=0\right], u(x, t, \varepsilon)$ has been evaluated and the corresponding numerical solution of (19) computed. The results for $x=2$ respecyively and $x=1$ are presented respectively in Fig. 2(a) and Fig. 2 (b). From the figures, it is clear that solution (32) compares well with the numerical solution.

For the under damped case, we consider $\rho=1, k=0.2, l=2, K=1$. The solutions of $(24)$ are $1.144465,2.543493,4.048082 \cdots$ or eigen-values are $-0.2 \pm 1.126854$, $-0.2 \pm 2.535618,-0.2 \pm 4.043138 \cdots$. For $\varepsilon=0.5$ and for initial values $[u(x, 0)=0.90667$ $\left.\sin (1.144465 x), u_{t}(x, 0)=0\right], u(x, t, \varepsilon)$ evaluated and the corresponding numerical solution of (19) has been computed. The results for $x=2$ and $x=1$ are presented in Fig. 3 (a) and Fig.3 (b) respectively. From the figures, it is clear that solution (32) compares well with the numerical solution.

When $k=2 \omega_{1}$, then solution Eq. (52) is useful for an over-damped solution of equation (19). We are interested to compare it with numerical solution (generated by finite difference method). Let us consider $\rho=1, k=1.3215, l=2, K=1$. The solutions of $(24)$ are1.144465, 2.543493, $4.048082 \cdots$ or eigen-values are $-1.3215 \pm 0.660728$, $-1.3215 \pm 2.173245 i,-1.3215 \pm 3.826304 i \cdots$ and one set of eigen-value is real. For $\varepsilon=0.5$ and initial values $\left[u(x, 0)=0.90667 \sin (1.144465 x), u_{t}(x, 0)=0\right], u(x, t, \varepsilon)$ has been evaluated and the corresponding numerical solution of (19) has been computed. The results for $x=2$ respectively and $x=1$ are presented respectively in Fig. 4(a) and Fig. 4(b). From the figures, it is clear that solution Eq. (52) compares well with the numerical solution.

\section{CONCLUSION}

A general formula is presented for obtaining the transient response of nonlinear systems governed by a hyperbolic-type partial differential equation with small nonlinearities. According to the unified theory $[4,5]$ there exists a general solution, used in three cases, i.e. over-damped, under-damped and un-damped. In previous papers $[5,7]$ only ordinary differential equations are considered. In the present paper, we observe a similar result for partial differential equations.

\section{ACKNOWLEDGEMENT}

The authors are grateful to two potential reviewers for their helpful comments/suggestions to prepare the revised manuscript. 


\section{REFERENCES}

1. N. N. Krylov and N. N. Bogoliubov, Introduction to Nonlinear Mechanics, Princeton University Press, New Jesey, 1943.

2. N. N. Bigoliubov and Yu. A. Mitropolskii, Asymptotic methods in the theory of nonlinear oncillations, Gordan and Breach, New York, 1961.

3. I. P. Popov, A generalization of the Bogoliubov asymptotic method in the theory of nonlinear oscillations (in Rusian), Dokl. Akad Nauk SSSR, 3 (1956), 308-310.

4. I. S. N. Murty, B. L. Deekshatulu and G. Krisna, General asymptotic method of KrylovBogoliubov for over-damped nonlinear systems, J. Frank Inst. 288 (1969) 49-64.

5. I. S. N. Murty, A univied Krylov-Bogoliubov method for solving second order nonlinear systems, Int. J. Nonlinear Mech. 6 (1971) 45-53.

6. G. N. Bojadziev and J. Edwards, On some asymptotic method for non-oscillatory and oscillatory processes, J. Nonlinear Vibration problems 20 (1981) 69-79.

7. M. Shamsul Alam, A unified Krylov-Bogoliubov-Mitropolskii method for solving $n$ - th order nonlinear system, J. Frank Inst. 339 (2002) 239-248.

8. M. Shamsul Alam, A unified Krylov-Bogoliubov-Mitropolskii method for solving $n$-th order nonlinear systems with varying coefficients, J. Sound and Vibration 265 (2003) 987-1002.

9. Yu. A. Mitropolskii and R. I. Mosencov, Lectures on the application of asymptotic methods of the solution of equations with partial derivatives, (in Russian), Ac. of Sci.Ukr. SSR, Kiev, 1968 .

10. G. N. Bojadziev and R, W. Lardner, Monofrequent oscillations in mechanical syste governed by second-order hyperbolic differential equation with small nonlinearities, Int. J. Nonlinear Mech. 8 (1973) 289-302.

11. G. N. Bojadziev and R. W. Lardner, Asymptotic solutions of partial differential equations with damping and delay, J. Quart. Appl. Math. 33 (1975) 205-214.

12. M. Shamsul Alam, M. Zahurul Islam and M. Ali Akber, A general form of Krylov- BogoliobovMitropolskii method for solving nonlinear partial differential equations, J. Sound and Vibration 285 (2005) 173-185.

13. M. Shamsul Alam, Asymptotic methods for second order over-damped and critically damped nonlinear systems, Soochow J. Math. 27 (2001) 187-200.

Received March 10,2008

\section{GIẢI HỆ PHUOONG TRİNH DAO HÀM RIÊNG PHI TUYÊN DẠG HYPERBOLIC BẰnG PHƯƠNG PHÁP KROLOV-BOGOLIUBOV-MITROPOLSKII}

Một nghiệm tiệm cận tổng quát được biểu diễn để khảo sát đặc trưng cửa hệ phi tuyến được mô hình bằng các phương. trình đạo hàm riêng dạng hyperbolic với hệ số phi tuyến bé. Phương pháp bao gồm tất cả các trường hợp khi các giá trị riêng của hệ không nhiễu loận tương ứng là thực, liên hợp phức, thuần ảo. Nó cho thấy bằng sự thay thế phù hợp của các giá trị riêng trong kết quả tổng quát, nghiệm tương ứng với mỗi trường hợp trong ba trường hợp là có thể thu được. Phương pháp này là một sự mở rộng của phương pháp Krolov-Bogoliubov-Mitropolskii, nó là sự phát triển ban đầu cho các trường hợp tắt dần, tắt dần chậm, tắt dần quá của phương trình đạo hàm thường bậc hai. Các phương pháp cũng bao gồm điều kiện đặc biệt của trường hợp tắt dần quá trong đó nghiệm tổng quát là không có nghĩa. 